\title{
G Se \\ S100A6 Promotes the Development and Apoptosis of Thyroid Cancer Through the PI3K/AKT/Mtor Pathway
}

\section{buran chen}

Wenzhou Medical University First Affiliated Hospital: The First Affiliated Hospital of Wenzhou Medical University

\section{Cuihua Shen}

Wenzhou Medical College First Affiliated Hospital: The First Affiliated Hospital of Wenzhou Medical University

\section{Danni Zheng}

Wenzhou Medical College First Affiliated Hospital: The First Affiliated Hospital of Wenzhou Medical University

\section{Yuhao Si}

Wenzhou Medical College First Affiliated Hospital: The First Affiliated Hospital of Wenzhou Medical University

\section{Chunlei Hu}

Wenzhou Medical College First Affiliated Hospital: The First Affiliated Hospital of Wenzhou Medical University

\section{Huihui li}

Wenzhou Medical University First Affiliated Hospital: The First Affiliated Hospital of Wenzhou Medical University

\section{Kaifu Tang}

Wenzhou Medical University First Affiliated Hospital: The First Affiliated Hospital of Wenzhou Medical University

\section{wei zhang ( $\nabla$ zhangwei2@wmu.edu.cn )}

Wenzhou Medical University First Affiliated Hospital: The First Affiliated Hospital of Wenzhou Medical University https://orcid.org/0000-0003-2316-270X

\section{Primary research}

Keywords: S100A6, thyroid cancer, proliferation, migration, biomarker, PI3K/AKT/mTOR pathway

Posted Date: June 8th, 2021

DOl: https://doi.org/10.21203/rs.3.rs-569422/v1 
License: (c) (i) This work is licensed under a Creative Commons Attribution 4.0 International License. Read Full License 


\section{Abstract}

\section{Background}

Thyroid cancer(TC) is the most common endocrine malignant tumor.High levels of S100A6 have been associated with progression in some type of human cancers, but its role in the molecular pathogenesis of these cancers is largely unknown. This study investigated the expression and functional roles of S100A6 in human thyroid cancer.

\section{Methods}

TCGA database and qRT-PCR were used to examine the expression of S100A6 in thyroid cancer. CCK-8 assay, colony formation assay, Transwell assay, invasion assay and western blot assay were used to identify the biological function of S100A6 in thyroid cancer cells. Flow cytometry is used to assess apoptosis.

\section{Results}

Compared with normal thyroid tissues, S100A6 is overexpressed in thyroid cancer tissues and cell lines. Silencing S100A6 can inhibit the proliferation, migration and invasion of TC cells, and induce their apoptosis.

\section{Conclusions}

S100A6 promotes the progression of thyroid cancer through PI3K/AKT/mTOR pathway and can be used as a target in the future treatment of thyroid cancer.

\section{Background}

Thyroid cancer (TC) is the most common endocrine tumor, and in the last decade its incidence has rapidly and sharply increased worldwide. Thyroid cancer ranks fifth among all cancers in females.[1] Most patients with thyroid cancer have a good prognosis after surgery, with a 10-year survival rate of > $90 \%$. However, some patients still experience recurrence and metastasis after treatment. The overall survival rate of patients with local recurrence is approximately $70-85 \%$, while the long-term survival rate of patients with distant metastasis is $30 \%-60 \%$.[2-4] Therefore, the mechanism of TC needs to be further studied.

S100A6 mainly exists in the cytoplasm, cell membrane, and nuclear envelope, including the inner surface of the nuclear membrane, and is a member of the S100A calcium-binding protein family. Several studies have shown that S100A6 is related to the occurrence and development of various tumors, including colon cancer, hepatocellular carcinoma, pancreatic cancer, and gastric cancer.[5-9] Therefore, we speculate that the abnormal expression of S100A6 is closely related to the proliferation and differentiation of tumor cells. 
Moreover, S100A6 mostly accumulates at the edge of the tumor, which may also be related to tumor invasion and migration. [10]However, its role in thyroid cancer remains unclear.

The PI3K/AKT/mTOR pathway is an important pathway in cancer development. [11]It regulates multiple processes, including cell survival, cell cycle progression, DNA repair, protein synthesis, glucose metabolism, angiogenesis, and cell migration. In this study,[12-15]

we verified, using real-time quantitative PCR, that S100A6 was highly expressed in tumor tissues; we then explored its function in thyroid cells by gene silencing and western blot analysis. The results indicated that S100A6 may be an important tumor-promoting gene in thyroid cancer through the activation of the $\mathrm{PISK} / \mathrm{AKT} / \mathrm{mTOR}$ pathway.

\section{Methods}

\section{Patients and thyroid tissue sample}

Thyroid cancer tissues (42 pairs) and corresponding normal tissues were collected from patients in the thyroid surgery department of the First Affiliated Hospital of Wenzhou Medical University, China, and stored in liquid nitrogen. Two pathologists performed the histological examinations of all specimens to confirm the histological diagnosis. All patients signed a letter of consent, and the patient's information was approved by the Ethics Committee of the First Affiliated Hospital of Wenzhou Medical University (approval no. 2012-57).

\section{Bioinformatics}

Information of 502 thyroid cancer patients with complete clinical case characteristics (age, sex, LNM, tumor size, clinical stage, etc.) was downloaded from the TCGA database for analysis. The public gene chip dataset was downloaded from the Gene Expression Omnibus (https://www.ncbi.nlm.nih.gov/geo/). The GSEA software (GSEA v3.0, http://www.broadinstitute.org/gsea) was used to perform a single GSEA between the low S100A6 and high S100A6 expression groups in TCGA.

\section{RNA isolation and real-time reverse transcription/polymerase chain reaction (qPCR)}

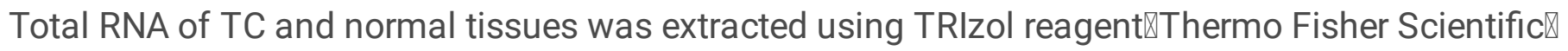
Waltham『USA『according to the manufacturer's instructions. CDNA synthesis was performed using the RerverTra Ace qPCR RT kit(Toyobo ,Osaka, Japan). The cDNA was stored at $-20^{\circ} \mathrm{C}$ for further processing. To detect the expression level of S100A6, qRT-PCR was performed using The Applied Biosystems 7500 Real-Time PCR system (Applied Biosystems; Thermo Fisher Scientific, Inc.).The primer sequences used

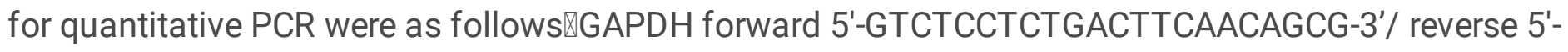
ACCACCCTGTTGCTGTAGCCAA-3' 'IS100A6 forward 5'-GAAATTGCAAGGCTGATGGAAG-3'/ reverse 5'GGCTTCATTGTAGATCAAAGCC-3'.

\section{Cell culture}


Human TC cell lines (FTC, KTC-1, and BCPAP) were provided by Professor Mingzhao Xing of Johns Hopkins University School of Medicine (Baltimore, Maryland, USA). The Htorti-3 cell line was purchased from the Stem Cell Bank, Chinese Academy of Sciences. TPC-1 and BCPAP cell lines were cultured in RPMI-1640 containing 10\% fetal bovine serum (FBS) (Gibco, Invitrogen, Carlsbad, CA, USA); FTC was cultured in DMEM medium (Gibco, Invitrogen, Carlsbad, CA, USA). Three cell lines were incubated at $37^{\circ} \mathrm{C}$ with $5 \% \mathrm{CO} 2$ in a standard cell culture incubator (Thermo Fisher Scientific).

Cell transfection

Thyroid cells were seeded in a six-well plate and transfected for $24 \mathrm{~h}$. S100A6 was silenced by siRNA using Lipofectamine RNAiMAX transfection reagent (Invitrogen, Grand Island, NY, USA). Both SiS100A6 and Si-NC were purchased from GenePharma (Shanghai, China). 48 hours after transfection, cells were subjected to the next experiment. The sequences were as follows: Si-S100A6, forward 5'AAGCTGCAGGATGCTGAAATT-3' ;Si-NC, forward 5-UUCUCCGAACGUGUCACGUTT-3 and reverse 5ACGUGACACGUUCGGAGAATT-3'.

\section{Cell proliferation and colony formation assay}

Cell Counting Kit-8 (Beyotime Biotechnology, Shanghai, China)and clone formation experiments were used to evaluate the proliferative ability of the cells. Transfected cells were spread (1000 cells per well) in a 96-well plate. Approximately $100 \mu \mathrm{L}$ of cck8 were added to each well, and the cells were incubated at 37 ${ }^{\circ} \mathrm{C}$ for $3 \mathrm{~h}$. The absorbance of each well at $450 \mathrm{~nm}$ was measured for four consecutive days.

\section{Clone formation experiment}

The transfected TC cells were seeded into a 6-well plate and incubated under the same conditions for 5-8 days. Cells were then fixed with $4 \%$ paraformaldehyde at room temperature for $15 \mathrm{~min}$, and stained with crystal violet. Pictures were taken under a strong light by a digital camera. Each experiment was repeated three times.

\section{Cell migration and invasion assays}

Transwell assays were performed to evaluate the migration and invasion capabilities of the cells. Transfected and control cells were collected using trypsin. The Transwell chamber (\#3422, Corning, NY, USA) and Matrigel Invasion Chamber (\#354480; Corning Biocat, New York, USA)was used in the assays. First, $600 \mu \mathrm{L}$ of medium containing $10 \%$ FBS were added to the lower chamber and the cells ( 35000 cells per well) were transferred to the upper chamber and incubated at $37^{\circ} \mathrm{C}$ for $20 \mathrm{~h}$. Cells migrating from the upper chamber to the lower chamber were fixed and stained with crystal violet for $15 \mathrm{~min}$. Three fields of view were randomly selected, and images were taken using a microscope at $\times 5$ magnification. Each experiment was repeated 3 times.

\section{Apoptosis analysis}


Annexin V-FITC apoptosis kit (\#556,547; Becton, Dickinson and Company, Franklin Lakes, NJ, USA) was used to detect cell apoptosis. Cells were collected, and stained with added Annexin V-FITC and PI in the dark for 15 min. The cells were analyzed using a flow cytometer (BD Biosciences, Franklin Lakes, USA). The apoptosis rate was equal to the percentage of late apoptosis (Q2) and the percentage of early apoptosis (Q3).

\section{Western blot analysis}

Total protein was extracted $48 \mathrm{~h}$ after transfection, and protein concentrations were determined using bicinchoninic acid assay (BCA). Protein lysates were separated by $10 \%$ SDS-PAGE electrophoresis and transferred to polyvinylidene fluoride (PVDF) membranes (EMD Millipore, Billerica, MA, USA). The PVDF membranes were blocked with skim milk for $2 \mathrm{~h}$ at room temperature and then washed three times with TBST. Cells were incubated with primary antibodies overnight at $4^{\circ} \mathrm{C}$. The primary antibodies are as follows:BCL-2(Abcam, Cambridge, Massachusetts, USA); S100A6, p-AKT, p-PI3K, p-MTOR, BAX and $\beta$ Actin (Proteintech, Wuhan, China). Membranes were rewashed with TBST three times and incubated with secondary antibodies (anti-mouse IgG or anti-rabbit lgG) for $1 \mathrm{~h}$ at room temperature. A chemiluminescence kit (Thermo Scientific, USA) was used to expose the films. Image Lab software was used to quantitatively analyze the bands.

\section{Statistical analysis}

Statistical analysis was performed using SPSS 23.0, and GraphPad Prism was used to create charts. Student's t-test or one-way ANOVA was used to assess differences between groups. Data in the figure are the mean \pm standard deviation. $P<0.05$, represents statistical significance.

\section{Results}

\section{S100A6 is highly expressed in thyroid cancer}

We downloaded transcriptome sequencing data from The Cancer Genome Atlas (TCGA) and GSE3678 to evaluate the expression of the S100A protein family in TC. Two data sets showed that s100a6 expression was significantly higher in tumor tissue than in normal thyroid tissue $(P<0.0001$ VFigure $1 A)$. In addition, we used the Tumor Immunity Estimation Resource (TIMER) database to perform a whole-cancer analysis of the expression of S100A6. Results showed that S100A6 was highly expressed in a variety of cancers (Figure 1B). In the TCGA database, compared with normal thyroid tissue, S100A6 is highly expressed in 502 pairs of thyroid cancer tissues $(P<0.0001$,Figure $2 A)$, and the same was observed in the GSE3678 dataset $(P<0.05$,Figure $2 B)$. In addition, we performed RNA sequencing on 70 pairs of thyroid cancer tissues and their paired adjacent tissues, and found the same phenomenon $(P<0.0001$,Figure $2 C)$. To verify this phenomenon, we used qRT-PCR to analyze 42 pairs of thyroid cancer tissues and corresponding adjacent tissues. As expected, S100A6 was overexpressed in the thyroid cancer tissues $(P<0.0001$,Figure 2D). 


\section{S100A6 expression level is related to the clinicopathological characteristics of thyroid cancer}

Next, we assessed the relationship between the expression level of S100A6 and clinical features in the TCGA database. According to the expression level of S100A6, patients were divided into two groups: a high-expression and low-expression group. In the TCGA cohort, higher S100A6 expression was associated with a higher T stage $(p<0.001)$, a greater incidence of lymph node metastasis $(L N M)(p<0.0001)$, a higher disease stage $(p<0.001)$, and a higher frequency of the classical type $(p<0.0001)$ (Table 1$)$. In addition, the expression of S100A6 was related to tumor size, LNM, and disease stage in the validated cohort (Table 2).We also found that most patients with higher disease stages had higher expression of S100A6 (Figure 2E-2G). Therefore, we believe that the clinicopathological characteristics of patients with thyroid cancer are related to the expression levels of S100A6.

\section{High expression of S100A6 increases the risk of lymph node metastasis in thyroid cancer}

Furthermore $₫$ we speculated that $\mathrm{S} 100 \mathrm{~A} 6$ may be the main risk factor for LNM in TC. Univariate logistic regression analysis showed that the expression levels of S100A6, age, disease stage, and histological type were related to the risk of LNM. Multivariate logistic regression analysis showed that these factors were also associated with LNM risk (Table 3).

\section{The significance of S100A6 expression in the diagnosis of patients with thyroid cancer}

To clarify the diagnostic value of S100A6, we performed an ROC curve analysis. In the TCGA database, the AUC was $84 \%(P<0.0001)$. Similarly, in the verification cohort and the GSE3678 database, the AUCs were $82.48 \%$ and $91.84 \%$, respectively (Figure $2 \mathrm{H}$ ). In addition, S100A6 also had diagnostic significance for T stage (Figure 2I). Therefore, we believe that the expression of S100A6 may be useful for the diagnosis of thyroid cancer.

\section{S100A6 promotes cell proliferation and clone formation}

We assessed the expression levels of S100A6 in normal cells and various thyroid cells by RT-qPCR and found that S100A6 was highly expressed in KTC-1, BCPAP, and FTC cell lines; therefore, these cell lines were selected for in vitro experiments (Figure 3A). First, we used siRNA to knockdown S100A6 in TC cell lines. RT-qPCR and western blotting revealed a reduction in the expression level of S100A6 in the three cell lines (Figure 3B,C). Then, we evaluated the effect of S100A6 on the proliferation of PTC cells. CCK-8 suggested that S100A6 knockdown reduced the proliferation of TC cells (Figure 3D,p<0.001). Clone formation experiments also proved this hypothesis (Figure 3E). Therefore, down-regulation of S100A6 inhibited the proliferation of PTC cell lines.

\section{Knockdown of S100A6 reduces the migration and invasion of thyroid cancer cells}

As mentioned above, since the expression of S100A6 is closely related to the LNM of TC, we used the Transwell assay and Matrigel invasion chamber assays to verify the effect of S100A6 on cell motility.Compared with the control group, the migration and invasion abilities of cells transfected with Si- 
S100A6 were significantly decreased $(P<0.001$,Figure $3 F, G)$. These results indicated that S100A6 promotes the migration and invasion of TC cells.

\section{S100A6 inhibits apoptosis and promotes the proliferation, migration and invasion of thyroid cancer cells by activating the PI3K/AKT/mTOR pathway}

Single gene set enrichment analysis (GSEA) was used to identify genes in TC that may be regulated by S100A6. The TC patients from TCGA were divided into two groups, S100A6 high expression group and S100A6 low expression group. The results showed that genes involved in apoptosis and related genes that activate the AKT pathway may be related to the expression of S100A6(Figure 4A-B). We used flow cytometry to detect cell apoptosis after transfection. After silencing S100A6, the apoptosis of KTC-1, BCPAP, and FTC cell lines increased, especially in early apoptotic cells (Figure 4C). Next, we quantified the protein levels of several representative markers of the AKT pathway and apoptosis. Activation of the $\mathrm{PI} 3 \mathrm{~K} / \mathrm{AKT} / \mathrm{mTOR}$ pathway is widely involved in the occurrence and development of thyroid cancer.[1618]We found that knockdown of S100A6 significantly reduced the expression of $p-m T O R, p-P I 3 K$, and $p$ AKT (Figure 4D). In addition, pro-apoptotic genes (Bax) and anti-apoptotic (Bcl-2) are representative markers of the apoptosis pathway. Down-regulation of S100A6 enhanced the expression of Bax and decreased the expression of Bcl-2. (Figure 4E).

\section{AKT activator SC79 can reverse the knockdown effect of Si-RNA}

To further confirm that S100A6 affects the occurrence and development of thyroid cancer through the $\mathrm{PI} 3 \mathrm{~K} / \mathrm{AKT} / \mathrm{mTOR}$ pathway, we conducted rescue experiments. SC79 is an AKT activator that can directly bind to AKT to activate AKT[19]. Using SC79 to treat transfected cells, and to perform functional tests, CCK-8 and colony formation experiments proved that transfected cells treated with SC79 had a stronger proliferation ability than simply transfected cells (Figure 5A,C). Transwell and Matrigel invasion chamber assays also showed that activated AKT could enhance the migration and proliferation of transfected cells (Figure 5D,E). Flow cytometry analysis showed that compared with Si-S100A6 group, SC79 inhibited cell apoptosis (Figure 5B).

\section{Discussion}

The incidence of thyroid cancer ranks fifth among women[1]. Although the prognosis is good, patients who relapse or have distant metastases still have a higher risk of death. For this reason, exploring the mechanism of the occurrence and development of thyroid cancer and identifying biomarkers is the aim of our research.

S100A6 is a calcium cellular peripheral protein that regulates cytoskeletal protein dynamics, cell proliferation, differentiation, calcium metabolism, ubiquitination, and acetylation. Previous studies have reported the high expression of [20-22]. S100A6 in several cancers. However, no studies have reported the mechanism of S100A6 in thyroid cancer. 
First, we consulted multiple public databases and found that the expression level of S100A6 was upregulated in cancer tissues. For further verification, we examined our validated cohort (42 pairs of thyroid cancer tissues and matched adjacent tissues). We found that the expression level of S100A6 was related to clinicopathological characteristics. In addition, the ROC curve also showed that S100A6 has diagnostic value. Through functional studies, we found that S100A6 promotes the proliferation, migration, and invasion of thyroid cells, and reduces their apoptosis. Western blotting assays proved that S100A6 promotes the occurrence of thyroid cancer through the PI3K/AKT/mTOR pathway and affects the expression of apoptotic geneBcl-2 and Bax. We verified this conclusion by using rescue experiments.

This study had several limitations. First, the results obtained were not verified through in vitro experiments. In addition, further studies are required to elucidate the mechanism by which S100A6 regulates the PI3K/AKT/mTOR pathway. In summary, we discovered that S100A6 is an effective oncogene, and can be a potential target for the diagnosis and treatment of thyroid cancer.

\section{Conclusion}

S100A6 promotes the proliferation, migration and invasion of TC cells, and inhibits their apoptosis. S100A6 promotes the progression of thyroid cancer through PI3K/AKT/mTOR pathway and can be used as a target in the future treatment of thyroid cancer.

\section{Abbreviations}

TC: Thyroid cancer; TCGA: The Cancer Genome Atlas; qRT-PCR: Quantitative

Real-time PCR; CCK-8: Cell Counting Kit-8; GSEA: Gene Set Enrichment Analysis; TIMER: Tumor Immunity Estimation Resource; LNM: lymph node metastasis.

\section{Declarations}

\section{Ethics approval and participation consent}

All patients signed a letter of consent, and the patient's information was approved by the Ethics Committee of the First Affiliated Hospital of Wenzhou Medical University (approval no. 2012-57).

\section{Consent for publication}

Not applicable.

\section{Availability of data and materials}

The sources of public datasets supporting the conclusions of this study are shown in this article. Other raw data are available on the main electronic data storage system of the First Affiliated Hospital of Wenzhou Medical University. 
Competing interests

The authors declare that they have no conflicts of interest.

Funding

None

Author's contribution

BRC is the first author and contributed to the research design. CHS and DNZ conducted a literature search. YHS got the data. CLH and HHL wrote this article. KFT and WZ revised the article and finally approved the version to be submitted. The final manuscript read and approved by all authors.

\section{Acknowledgments}

The author would like to thank the researchers and staff who contributed to this research.

\section{References}

1. Siegel RL, Miller KD, Jemal A: Cancer statistics, 2020. Ca-a Cancer Journal for Clinicians 2020, 70(1):7-30.

2. Haq M, Harmer C: Differentiated thyroid carcinoma with distant metastases at presentation: prognostic factors and outcome. Clinical Endocrinology 2005, 63(1):87-93.

3. Sampson E, Brierley JD, Le LW, Rotstein L, Tsang RW: Clinical management and outcome of papillary and follicular (differentiated) thyroid cancer presenting with distant metastasis at diagnosis. Cancer 2007, 110(7):1451-1456.

4. Shaha AR, Shah JP, Loree TR: Differentiated thyroid cancer presenting initially with distant metastasis. American journal of surgery 1997, 174(5):474-476.

5. Chen X, Liu X, Lang H, Zhang S, Luo Y, Zhang J: S100 Calcium-Binding Protein A6 Promotes Epithelial-Mesenchymal Transition through beta-Catenin in Pancreatic Cancer Cell Line. Plos One 2015, 10(3).

6. Duan L, Wu R, Zou Z, Wang H, Ye L, Li H, Yuan S, Li X, Zha H, Sun H et al: S100A6 stimulates proliferation and migration of colorectal carcinoma cells through activation of the MAPK pathways. International Journal of Oncology 2014, 44(3):781-790.

7. Li Y, Wagner ER, Yan Z, Wang Z, Luther G, Jiang W, Ye J, Wei Q, Wang J, Zhao L et al: The CalciumBinding Protein S100A6 Accelerates Human Osteosarcoma Growth by Promoting Cell Proliferation and Inhibiting Osteogenic Differentiation. Cellular Physiology and Biochemistry 2015, 37(6):23752392.

8. Li Z, Tang M, Ling B, Liu S, Zheng Y, Nie C, Yuan Z, Zhou L, Guo G, Tong A et al: Increased expression of S100A6 promotes cell proliferation and migration in human hepatocellular carcinoma. Journal of 
Molecular Medicine-Jmm 2014, 92(3):291-303.

9. Wang X-H, Zhang L-H, Zhong X-Y, Xing X-F, Liu Y-Q, Niu Z-J, Peng Y, Du H, Zhang G-G, Hu Y et al: S100A6 Overexpression Is Associated with Poor Prognosis and Is Epigenetically Up-Regulated in Gastric Cancer. American Journal of Pathology 2010, 177(2):586-597.

10. Ilg EC, Schafer BW, Heizmann CW: Expression pattern of S100 calcium-binding proteins in human tumors. International journal of cancer 1996, 68(3):325-332.

11. Robbins HL, Hague A: The PI3KJAkt Pathway in Tumors of endocrine Tissues. Frontiers in Endocrinology 2016, 6.

12. Saji M, Vasko V, Kada F, Allbritton EH, Burman KD, Ringel MD: Akt1 contains a functional leucine-rich nuclear export sequence. Biochemical and Biophysical Research Communications 2005, 332(1):167173.

13. Hers I, Vincent EE, Tavare JM: Akt signalling in health and disease. Cellular Signalling 2011, 23(10):1515-1527.

14. Martelli AM, Tabellini G, Bressanin D, Ognibene A, Goto K, Cocco L, Evangelisti C: The emerging multiple roles of nuclear Akt. Biochimica Et Biophysica Acta-Molecular Cell Research 2012, 1823(12):2168-2178.

15. Ocana A, Vera-Badillo F, Al-Mubarak M, Templeton AJ, Corrales-Sanchez V, Diez-Gonzalez L, CuencaLopez MD, Seruga B, Pandiella A, Amir E: Activation of the PI3K/mTOR/AKT Pathway and Survival in Solid Tumors: Systematic Review and Meta-Analysis. Plos One 2014, 9(4).

16. Campos M, Kool MMJ, Daminet S, Ducatelle R, Rutteman G, Kooistra HS, Galac S, Mol JA: Upregulation of the PI3K/Akt Pathway in the Tumorigenesis of Canine Thyroid Carcinoma. Journal of Veterinary Internal Medicine 2014, 28(6):1814-1823.

17. Hou P, Liu D, Shan Y, Hu S, Studeman K, Condouris S, Wang Y, Trink A, El-Naggar AK, Tallini G et al: Genetic alterations and their relationship in the phosphatidylinositol 3-kinase/Akt pathway in thyroid cancer. Clinical Cancer Research 2007, 13(4):1161-1170.

18. Santarpia L, El-Naggar AK, Cote GJ, Myers JN, Sherman SI: Phosphatidylinositol 3-Kinase/Akt and Ras/RafMitogen-Activated protein kinase pathway mutations in anaplastic thyroid cancer. Journal of Clinical Endocrinology \& Metabolism 2008, 93(1):278-284.

19. Jo H, Mondal S, Tan D, Nagata E, Takizawa S, Sharma AK, Hou Q, Shanmugasundaram K, Prasad A, Tung JK et al: Small molecule-induced cytosolic activation of protein kinase Akt rescues ischemiaelicited neuronal death. Proceedings of the National Academy of Sciences of the United States of America 2012, 109(26):10581-10586.

20. Cross SS, Hamdy FC, Deloulme JC, Rehman I: Expression of S100 proteins in normal human tissues and common cancers using tissue microarrays: S100A6, S100A8, S100A9 and S100A11 are all overexpressed in common cancers. Histopathology 2005, 46(3):256-269.

21. Tsoporis JN, Izhar S, Parker TG: Expression of S100A6 in Cardiac Myocytes Limits Apoptosis Induced by Tumor Necrosis Factor-alpha. Journal of Biological Chemistry 2008, 283(44):3017430183. 
22. Ishii A, Suzuki M, Satomi K, Kobayashi H, Sakashita S, Kano J, Pei Y, Minami Y, Ishikawa S, Noguchi $\mathrm{M}$ : Increased cytoplasmic S100A6 expression is associated with pulmonary adenocarcinoma progression. Pathology International 2009, 59(9):623-630.

\section{Tables}

Table 1. The association between S100A6 expression and clinicopathological features in the TCGA cohort 


\begin{tabular}{|c|c|c|c|c|}
\hline Factors & Patients & High expression & Low expression & $p$ \\
\hline \multicolumn{5}{|l|}{ Gender } \\
\hline Female & 367 & 178 & 189 & 0.314 \\
\hline Male & 135 & 73 & 62 & \\
\hline \multicolumn{5}{|l|}{ Age (years) } \\
\hline$<60$ & 389 & 199 & 190 & 0.393 \\
\hline$\geq 60$ & 113 & 52 & 61 & \\
\hline \multicolumn{5}{|l|}{ T stage } \\
\hline$|+| \mid$ & 308 & 131 & 177 & $<0.001^{\star}$ \\
\hline III+IV & 193 & 120 & 73 & \\
\hline \multicolumn{5}{|l|}{$\mathrm{N}$ stage } \\
\hline NO & 280 & 107 & 173 & $<0.001^{\star}$ \\
\hline $\mathrm{Nx}$ & 222 & 144 & 78 & \\
\hline \multicolumn{5}{|l|}{ M stage } \\
\hline MO & 283 & 147 & 186 & 0.504 \\
\hline Mx & 9 & 6 & 3 & \\
\hline \multicolumn{5}{|c|}{ Disease stage } \\
\hline$|+| \mid$ & 333 & 147 & 186 & $<0.001^{\star}$ \\
\hline III+IV & 167 & 103 & 64 & \\
\hline \multicolumn{5}{|c|}{ Neoplasm focus type } \\
\hline Unifocal & 276 & 143 & 133 & 0.419 \\
\hline Multifocal & 226 & 108 & 118 & \\
\hline \multicolumn{5}{|l|}{ Histological } \\
\hline Classical & 356 & 205 & 151 & $<0.001^{\star}$ \\
\hline Others & 146 & 46 & 100 & \\
\hline \multicolumn{5}{|l|}{ New event } \\
\hline No & 458 & 224 & 234 & 0.155 \\
\hline Yes & 44 & 27 & 17 & \\
\hline
\end{tabular}

Abbreviations: S100A6, S100 calcium-binding protein 1; TCGA, The Cancer Genome Atlas; 
Table 2. The correlation between S100A6 expression and clinicopathological factors in the validated cohort

\begin{tabular}{|c|c|c|c|c|}
\hline Factors & Patients & High expression (\%) & Low expression (\%) & $p$ \\
\hline \multicolumn{5}{|l|}{ Age (years) } \\
\hline$\geq 55$ & 15 & 9 & 6 & 0.334 \\
\hline$<55$ & 27 & 12 & 15 & \\
\hline \multicolumn{5}{|c|}{ Gender (mm) } \\
\hline female & 23 & 10 & 13 & 0.800 \\
\hline male & 19 & 9 & 10 & \\
\hline \multicolumn{5}{|c|}{ Lymph node metastasis } \\
\hline No & 19 & 4 & 15 & $0.004 *$ \\
\hline Yes & 23 & 15 & 8 & \\
\hline \multicolumn{5}{|l|}{ Tumor size } \\
\hline$\geq 10$ & 25 & 18 & 7 & $0.002 *$ \\
\hline$<10$ & 17 & 4 & 13 & \\
\hline \multicolumn{5}{|c|}{ Neoplasm focus type } \\
\hline Unifocal & 34 & 17 & 17 & 0.524 \\
\hline Multifocal & 8 & 3 & 5 & \\
\hline \multicolumn{5}{|c|}{ Disease stage (AJCC7) } \\
\hline$|+| \mid$ & 24 & 10 & 14 & $0.027 *$ \\
\hline III+IV & 14 & 11 & 3 & \\
\hline
\end{tabular}

Table 3. Univariate and multivariate COX regression analysis for overall survival in the TCGA cohort 


\begin{tabular}{|c|c|c|c|c|c|c|}
\hline \multirow[t]{2}{*}{ Parameters } & \multicolumn{3}{|c|}{ Univariate analysis } & \multicolumn{3}{|c|}{ Multivariate analysis } \\
\hline & HR & $95 \% \mathrm{Cl}$ & $p$ & $\mathrm{HR}$ & $95 \% \mathrm{Cl}$ & $p$ \\
\hline $\begin{array}{l}\text { S100A6 expression (high vs. } \\
\text { low) }\end{array}$ & 2.126 & $\begin{array}{l}1.410- \\
3.207\end{array}$ & $<0.001^{\star}$ & 0.468 & $\begin{array}{l}0.314- \\
0.697\end{array}$ & $<0.001^{*}$ \\
\hline $\begin{array}{l}\text { Gender } \\
\text { (female vs.male) }\end{array}$ & 0.695 & $\begin{array}{l}0.438- \\
1.104\end{array}$ & 0.123 & & & \\
\hline $\begin{array}{l}\text { Age (years) } \\
(\geq 60 \text { vs. }<60)\end{array}$ & 0.288 & $\begin{array}{l}0.159- \\
0.523\end{array}$ & $<0.001^{\star}$ & 0.31 & $\begin{array}{l}0.178- \\
0.538\end{array}$ & $<0.001^{*}$ \\
\hline $\begin{array}{l}\text { T stage } \\
(\mathrm{T} 1+\mathrm{T} 2 \text { vsT3+T4) }\end{array}$ & 1.476 & $\begin{array}{l}0.919- \\
2.371\end{array}$ & 0.107 & & & \\
\hline Disease Stag(I+|l vs. III+IV) & 4.459 & $\begin{array}{l}2.590- \\
7.676\end{array}$ & $<0.001^{\star}$ & 5.631 & $\begin{array}{l}3.465- \\
9.153\end{array}$ & $<0.001$ \\
\hline $\begin{array}{l}\text { Histological type } \\
\text { (classical vs. others) }\end{array}$ & 3.014 & $\begin{array}{l}1.828- \\
4.970\end{array}$ & $<0.001^{\star}$ & 2.75 & $\begin{array}{l}1.732- \\
4.512\end{array}$ & $<0.001$ \\
\hline $\begin{array}{l}\text { Neoplasm focus type } \\
\text { (Unifocal vs. Multifocal) }\end{array}$ & 1.491 & $0.982-2.64$ & 0.061 & & & \\
\hline $\begin{array}{l}\text { Disease Stage } \\
(I+I \mid \text { vs. III+IV) }\end{array}$ & 4.459 & $\begin{array}{l}2.590- \\
7.676\end{array}$ & $<0.001^{\star}$ & 5.631 & $\begin{array}{l}3.465- \\
9.153\end{array}$ & $<0.001$ \\
\hline
\end{tabular}

Abbreviations: TCGA, The Cancer Genome Atlas; S100A6, S100 calcium-binding protein 1; HR: Hazard ratio; Cl: Confidence interval.

\section{Figures}


A

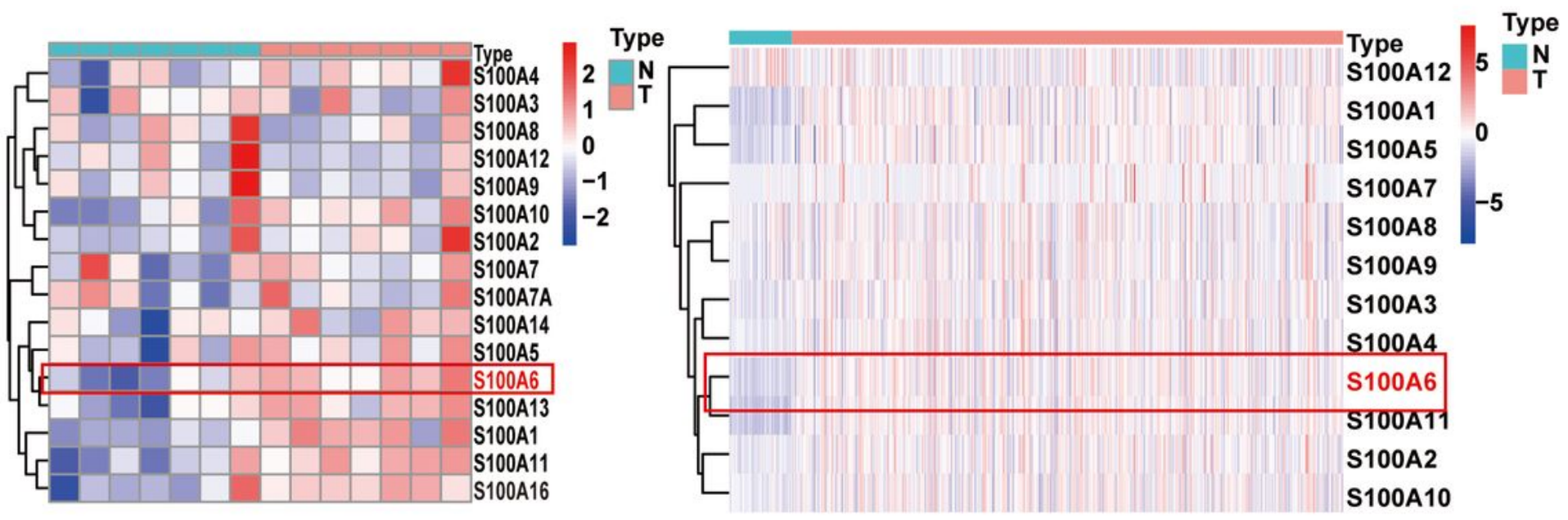

B
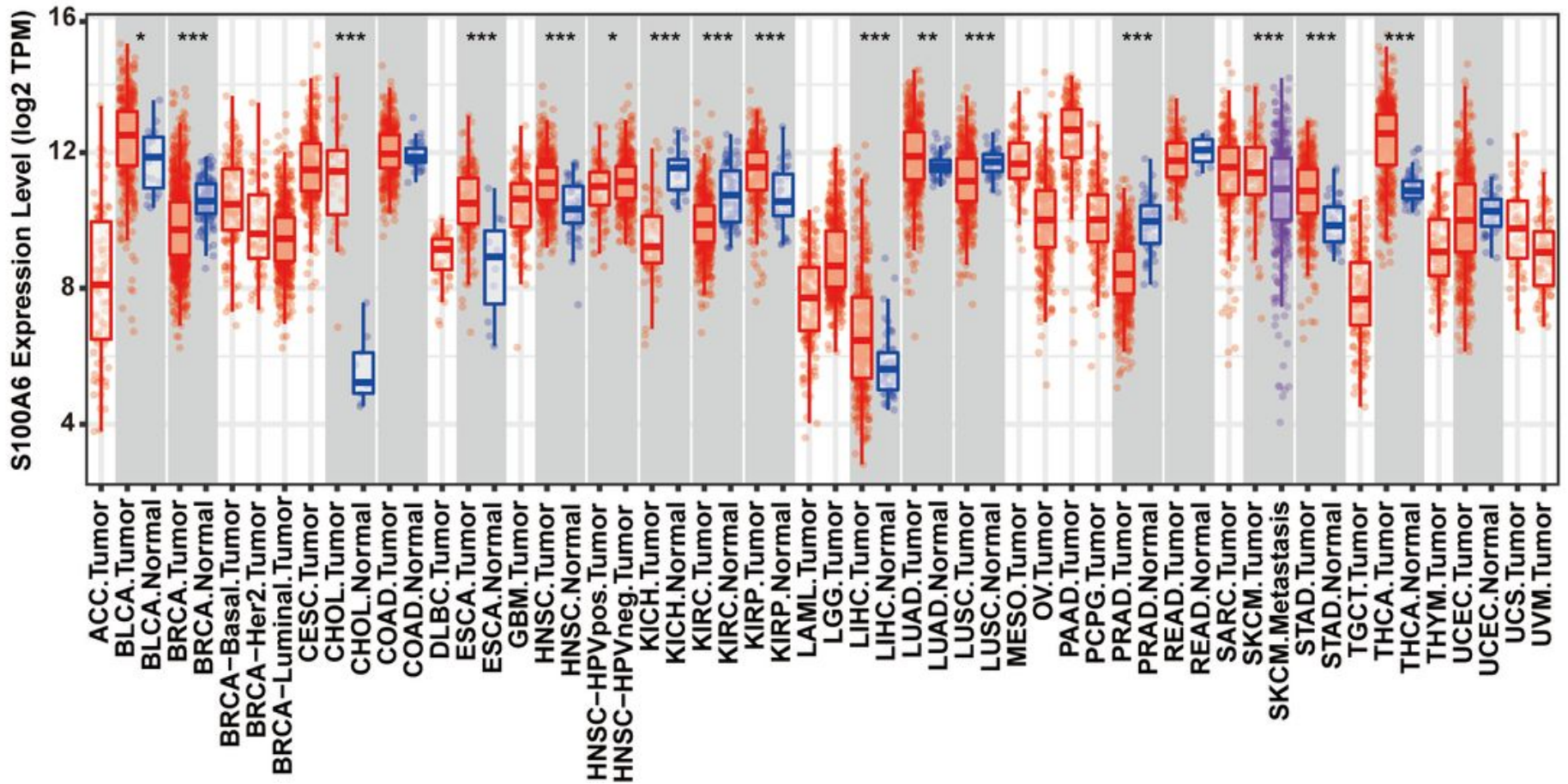

Figure 1

S100A6 is upregulated in thyroid cancer (A) Analysis of the expression level of the $S 100$ family in the TCGA database and GSE 3678. (B) S100A6 is overexpressed in a variety of cancers in TCGA. The Wilcoxon test was used to assess the statistical significance of the differential expression. ${ }^{\star} \mathrm{p}<0.05,{ }^{* \star} \mathrm{p}<0.01,{ }^{\star \star *} \mathrm{p}<0.001$. 
A
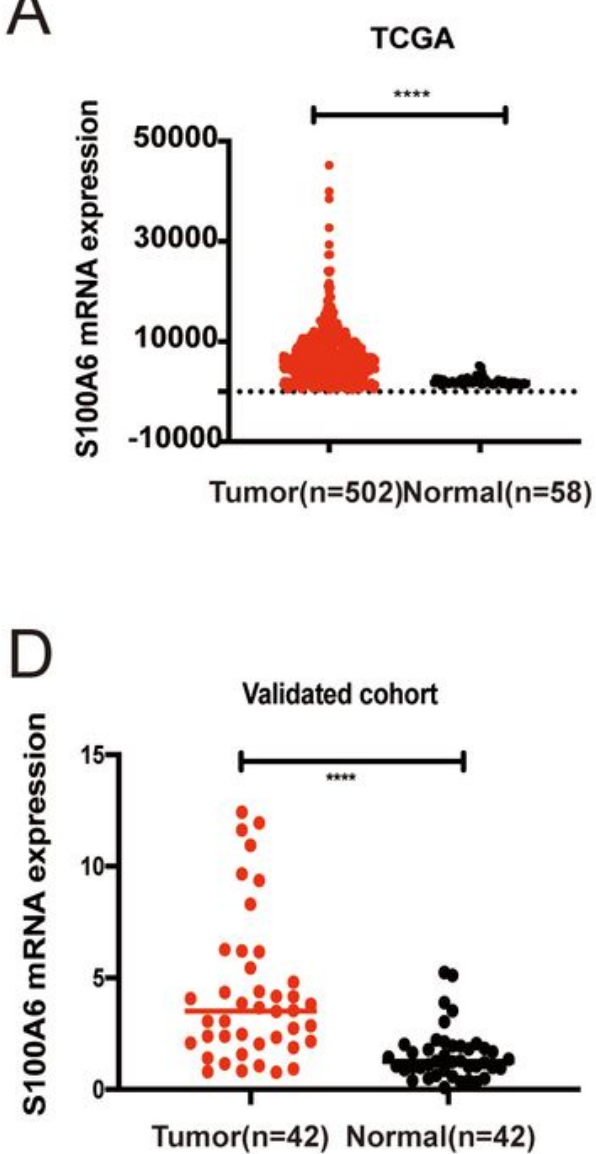

G

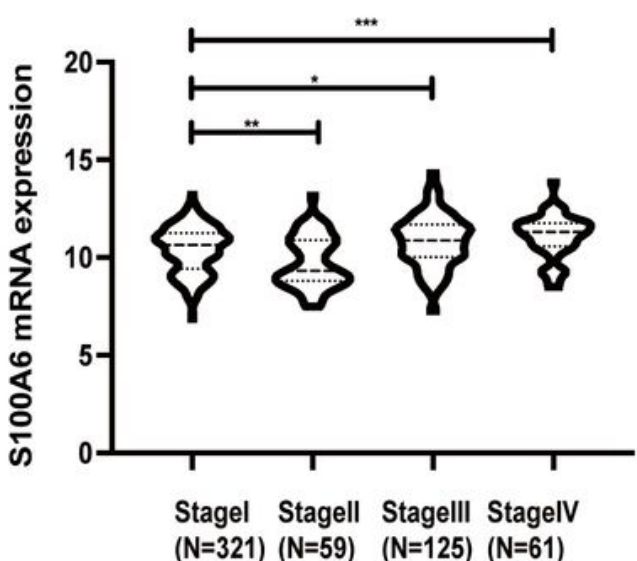

GSE3678

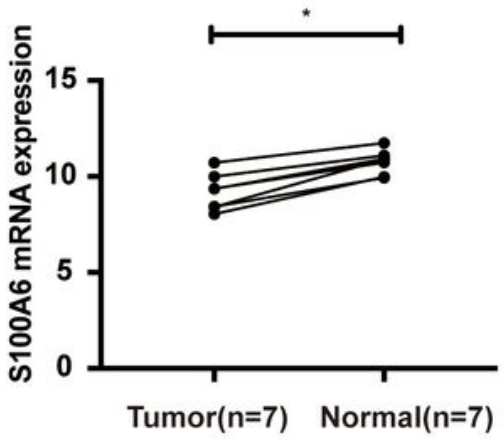

E

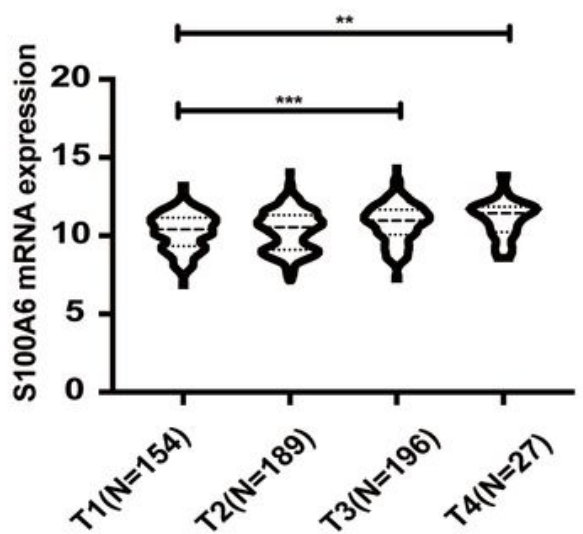

$\mathrm{H}$

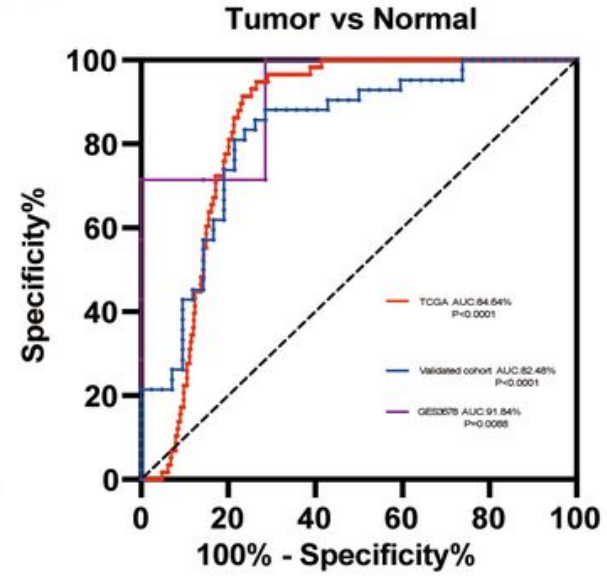

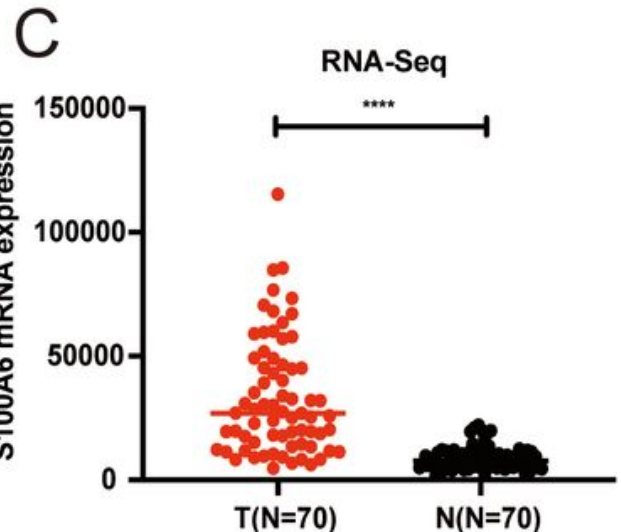

$\mathrm{F}$

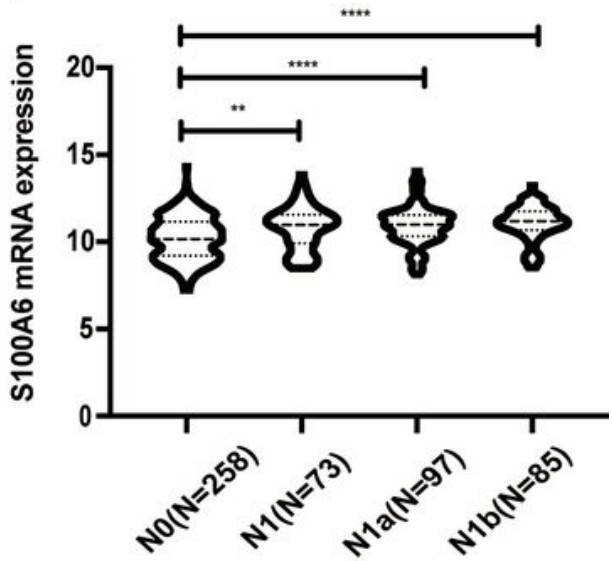

$\mathrm{Tl}+\mathrm{T} 2$ vs $\mathrm{T} 3+\mathrm{T} 4$

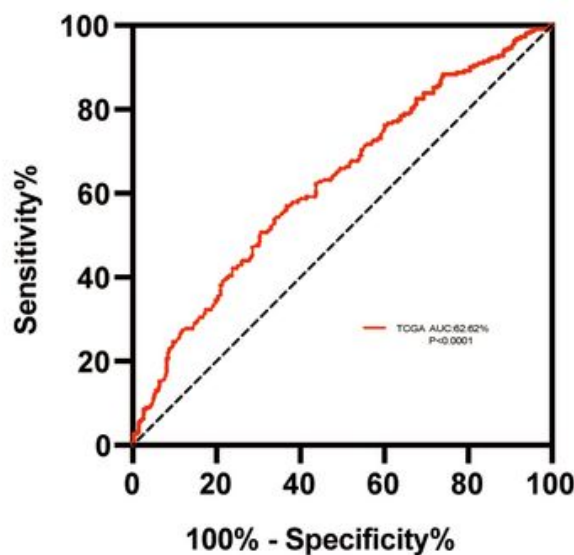

Figure 2

S100A6 is highly expressed in TC (A-B) In the TCGA and GSE3678 database, the expression level of S100A6 in TC tissues was higher than that in normal tissues. (C) S100A6 was highly expressed in tumor tissues among 70 pairs of RNA sequencing tissues. (D) S100A6 was overexpressed in tumor tissues in the validation cohort using qRT-PCR. (E-G) Different $\mathrm{T}, \mathrm{N}$, and disease stage show that the expression level of S100A6 is different. Higher S100A6 expression is often associated with poorer clinicopathological 
characteristics $(\mathrm{H})$ In the TCGA and GSE3678 database, and verification cohort, the ROC curve shows that S100A6 has diagnostic value. (I) In TCGA, the diagnostic significance of S100A6 expression levels at different T stages. Statistical analysis: (A) Mann-Whitney test (B) Paired t-test (C-D) Wilcoxon test (E-G) Kruskal-Wallis test ${ }^{\star} \mathrm{p}<0.05,{ }^{* \star} \mathrm{p}<0.01,{ }^{* \star *} \mathrm{p}<0.001,{ }^{* \star * *} \mathrm{p}<0.0001$.
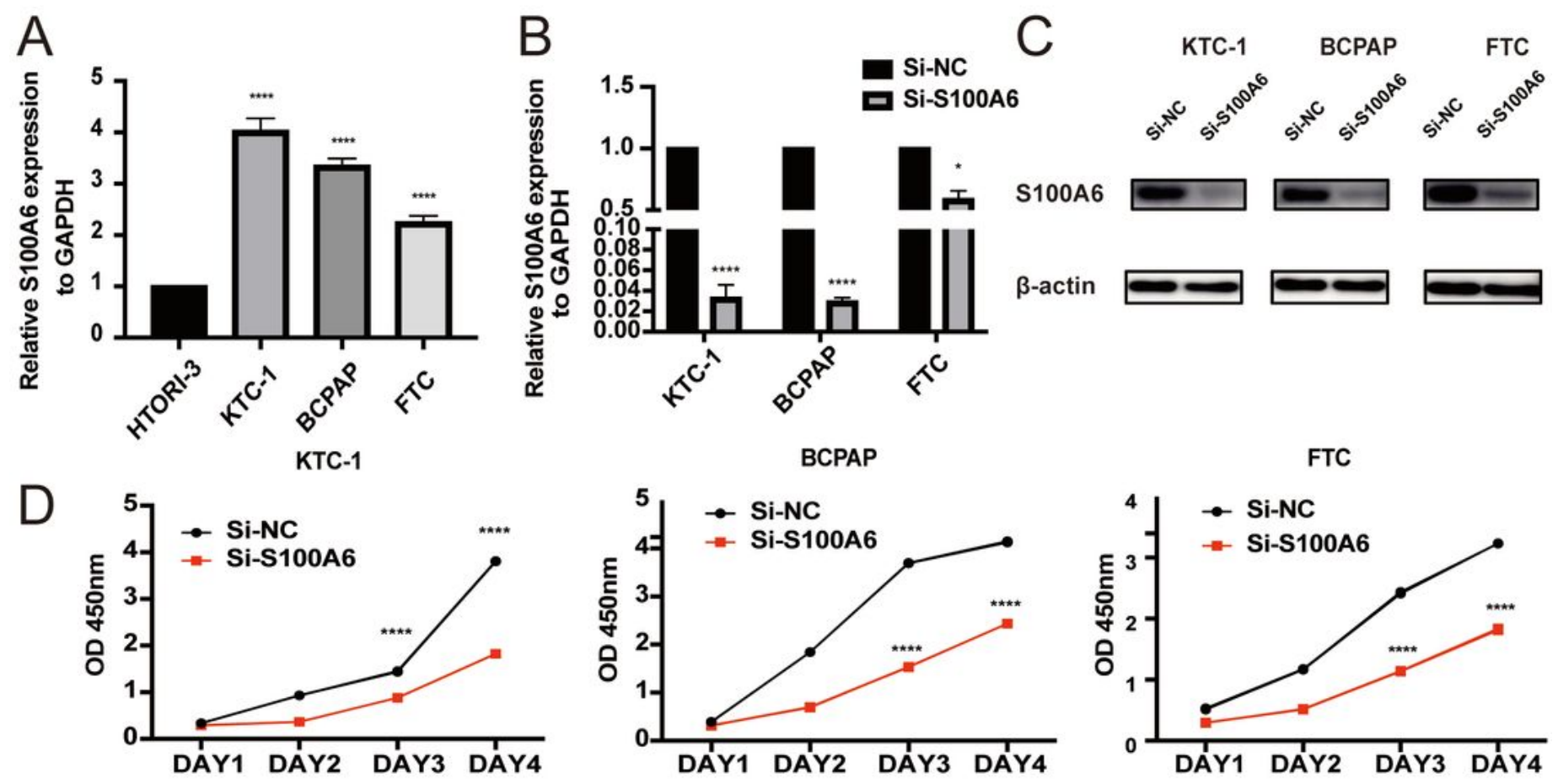

E
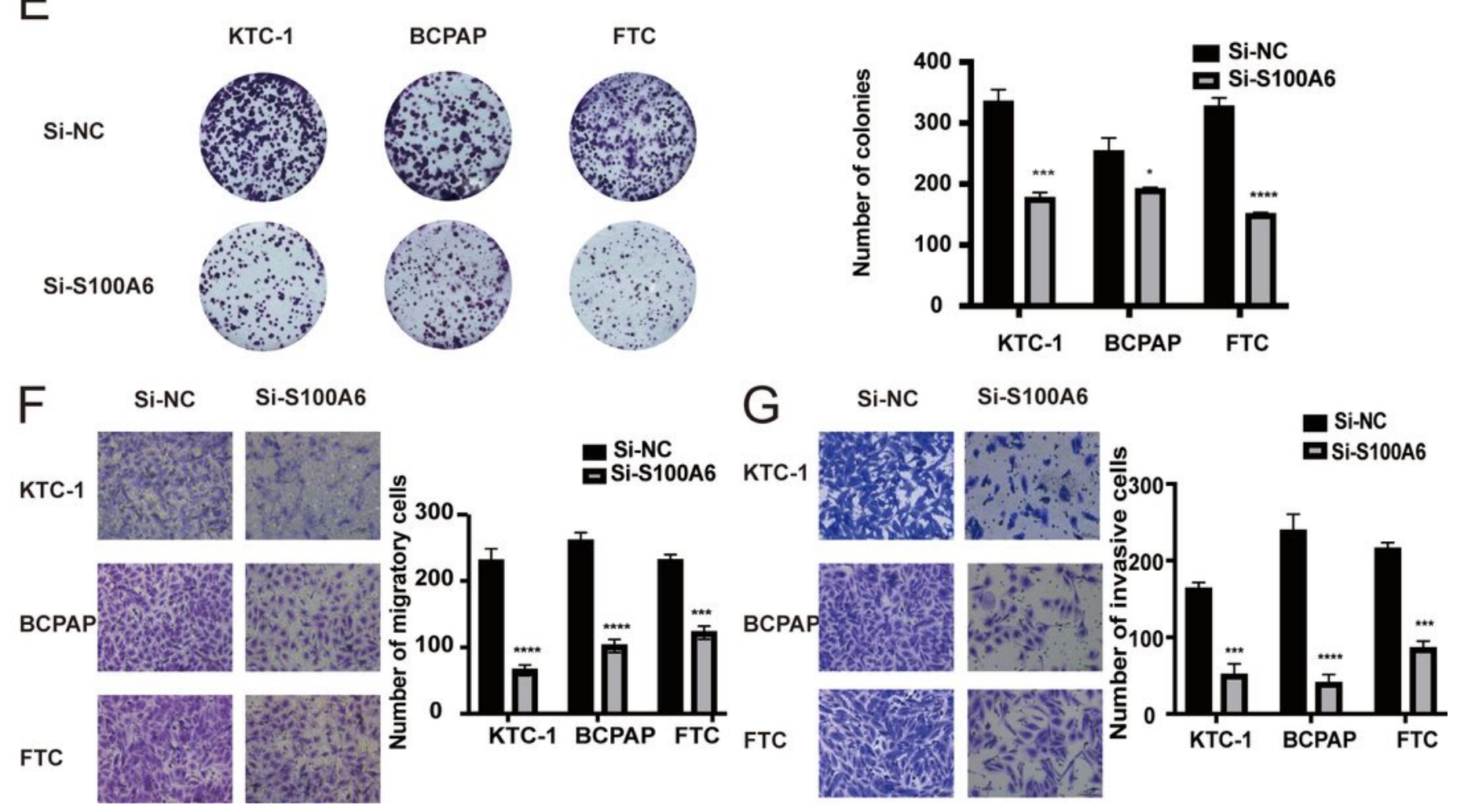

Figure 3 
S100A6 is highly expressed in thyroid cell lines and promotes its proliferation, migration and invasion. (A)Compared with the normal thyroid cell line HTORI-3, S100A6 was relatively highly expressed in the TC cell line. (B) In the three cell lines, the expression level of S100A6 in the Si-S100A6 group was lower than that in the Si-NC group. (C) In the Si-S100A6 group, the S100A6 protein expression level was lower than that of the Si-NC group. (D-E) CKK-8 analysis and clone formation experiments in three TC lines.(F-G) Migration and invasion experiments were performed on the Si-NC and Si-S100A6 groups; 3 fields of view were randomly selected for analysis and measurement under the microscope. Statistical analysis:

(D)Two-way analysis of variance (A, B, C, E, F, G) Student's t-test. ${ }^{\star} p<0.05,{ }^{\star \star} p<0.01$, ${ }^{\star \star \star} p<0.001 \mathrm{D}$ $\star \star \star \star x p<0.0001$. 
A

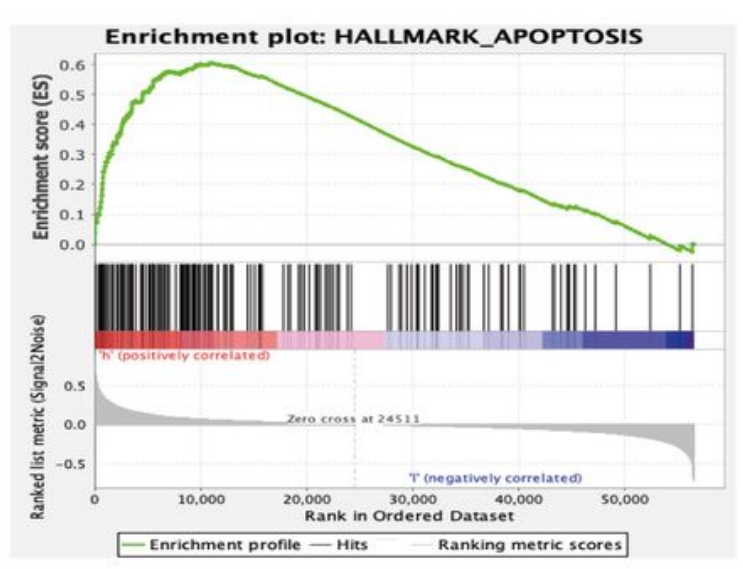

B

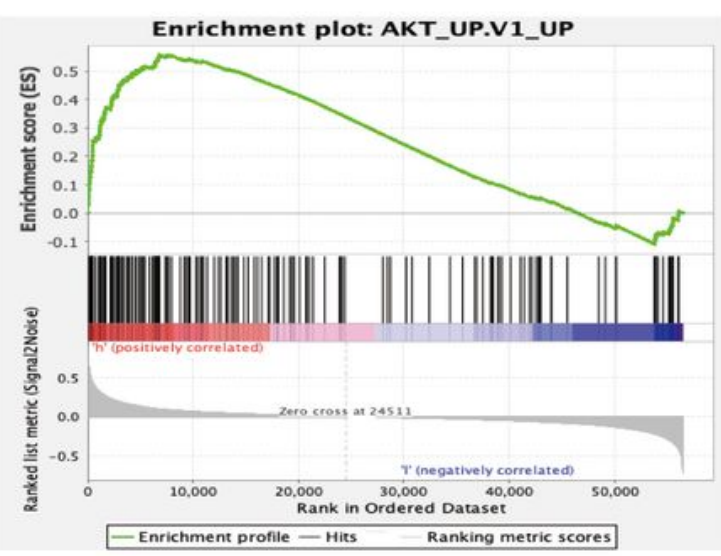

KTC-1

BCPAP

FTC
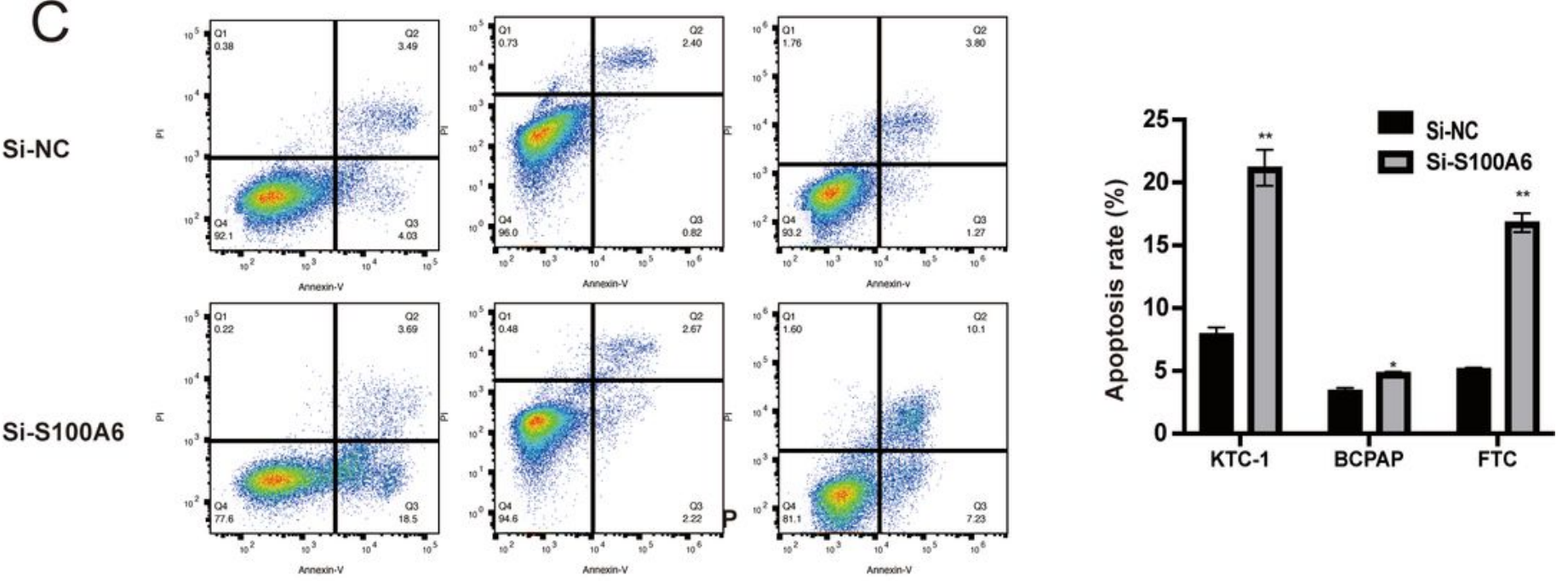

D

KTC-1

BCPAP

FTC
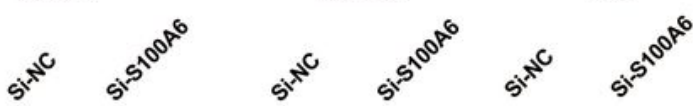

E
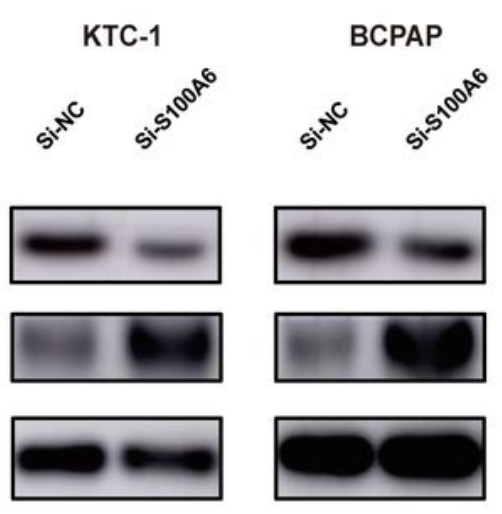

FTC

p-mTOR
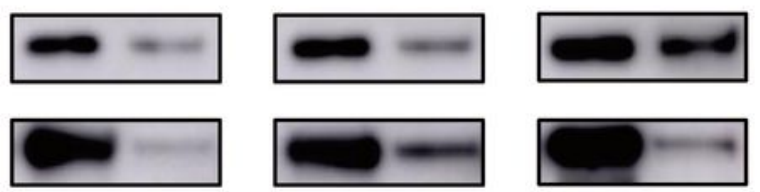

BCL-2

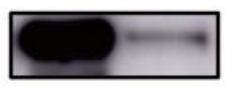

Bax

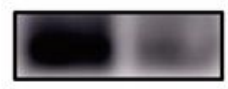

$\beta$-actin
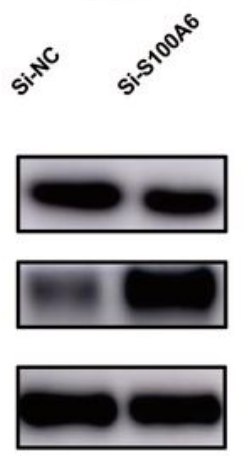

\section{Figure 4}

S100A6 promotes the development of thyroid cancer through the PI3K/AKT/mTOR pathway while inhibiting its apoptosis. (A-B)A single GSEA based on TCGA data indicates that S100A6 is associated with apoptosis and the AKT pathway. (C) Silencing S100A6 promotes the apoptosis of TC cells. At protein level, down-regulation of S100A6 increased BAX expression and decreased the expression of Bcl2. (D) After silencing S100A6, p-mTOR, p-PI3K and p-AKT were down-regulated at the protein level. (E) 
Western blot analysis showed that at the protein level, down-regulation of S100A6 can increase the expression of Bax and decrease the expression of Bcl-2. Statistical analysis: (C) Student's t-test. ${ }^{*} p<0.05,{ }^{* \star} p<0.01$.

A

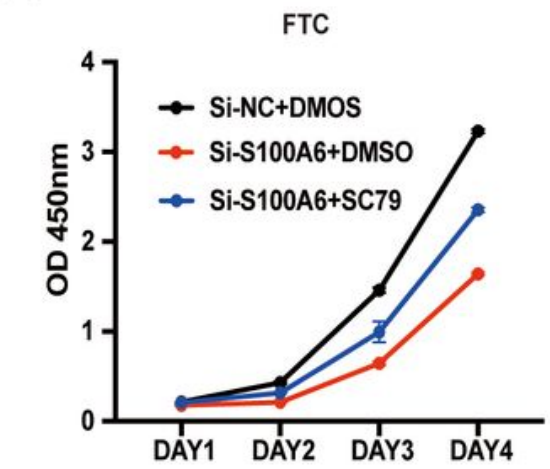

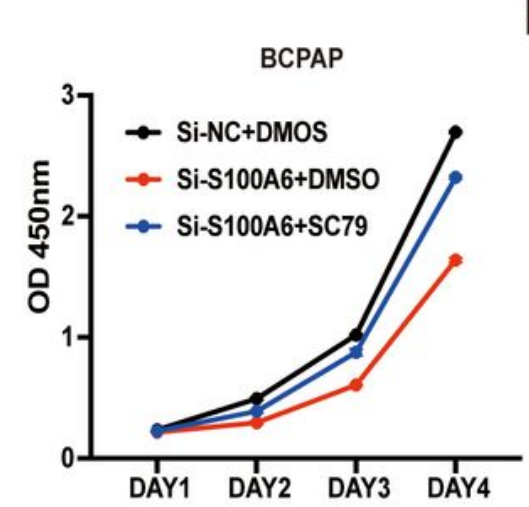

C

Si-NC+DMSO
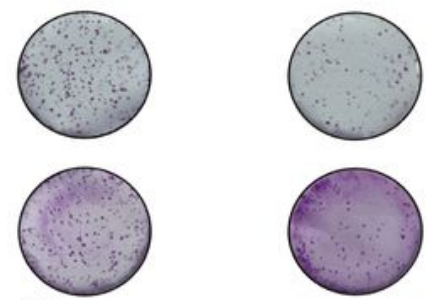

BCPAP
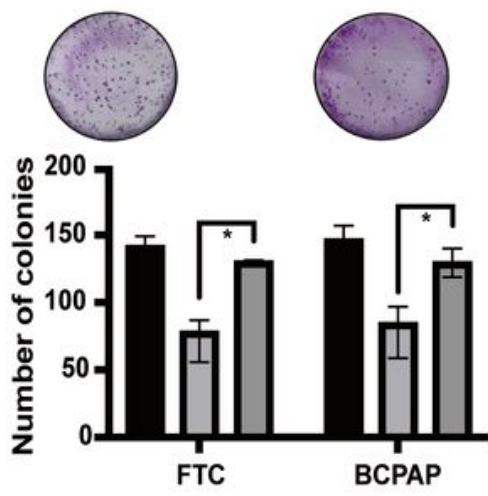

Si-NC+DMOS Si-S100A6+DMSO Si-S100A6+SC79

FTC
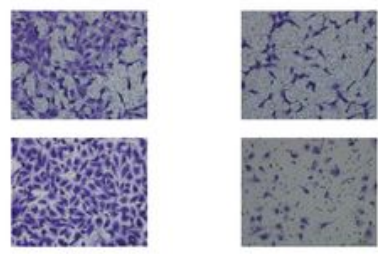

BCPAP

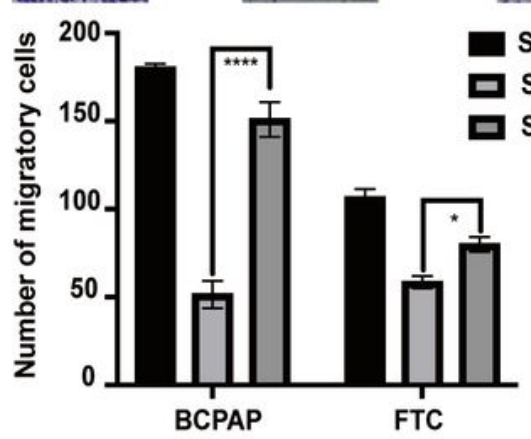

Si-NC+DMOS
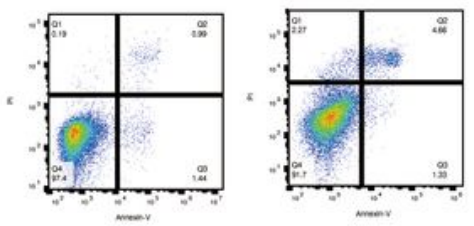

Si-S100A6+DMSO
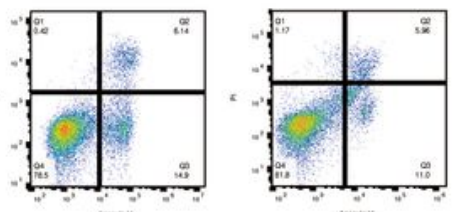

Si-S100A6+SC79
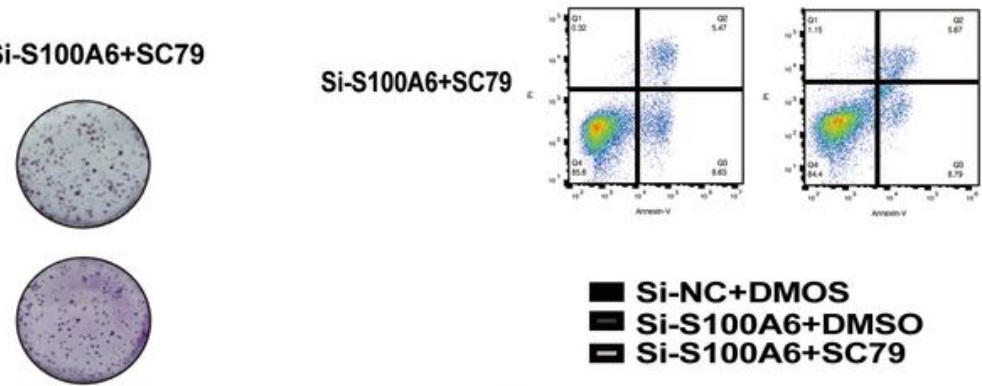

Si-NC+DMOS

Si-S100A6+DMSO

모-S100A6+SC79

E

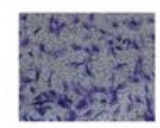

Si-NC+DMOS

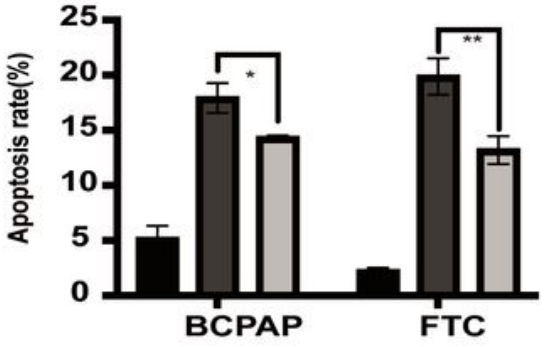

FTC

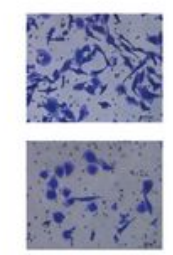

Si-NC+DMOS

Si-S100A6+DMSO

Si-S100A6+SC79

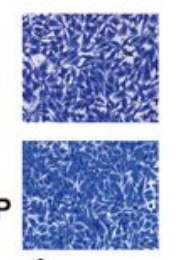

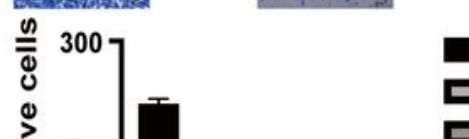

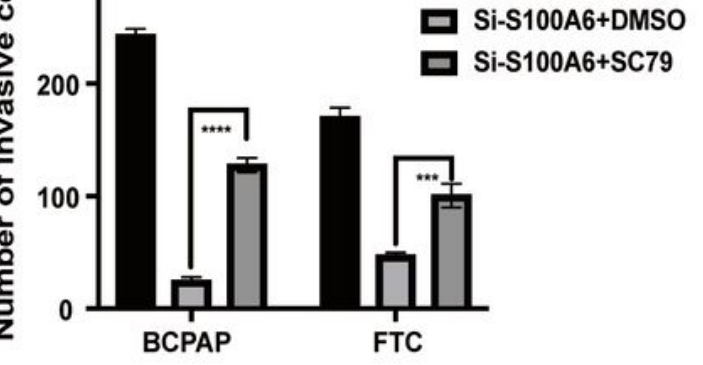

Figure 5

AKT activator SC79 can reverse the knockdown effect of Si-RNA $(A, C)$ CCK-8 and clone formation experiments were performed on the three groups Si-NC+DMSO, Si-S100A6+DMSO, and Si-S100A6+SC79. 
(B) Compared with the Si-S100A6+DMSO group, SC79 promotes cell apoptosis. (D-E) Transwell migration and Matrigel invasion assays were performed on three groups, namely: Si-NC+DMSO, Si-S100A6+DMSO, and Si-S100A6+SC79. Statistical analysis: Two-way analysis of multiple comparisons(A, B, C, D, E).

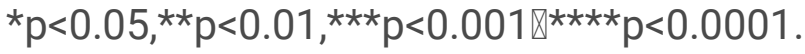

